\title{
Scaling invariance of the diffusion coefficient in a family of two-dimensional Hamiltonian mappings
}

\author{
Juliano A. de Oliveira, ${ }^{1,2}$ Carl P. Dettmann, ${ }^{3}$ Diogo R. da Costa, ${ }^{3,4}$ and Edson D. Leonel ${ }^{1}$ \\ ${ }^{1}$ Departamento de Física, UNESP, Universidade Estadual Paulista, Avenida 24A, 1515 13506-900, Rio Claro, São Paulo, Brazil \\ ${ }^{2}$ UNESP, Universidade Estadual Paulista, Câmpus São João da Boa Vista, São João da Boa Vista, São Paulo, Brazil \\ ${ }^{3}$ School of Mathematics, University of Bristol, Bristol BS8 1TW, United Kingdom \\ ${ }^{4}$ Instituto de Física da USP, Cidade Universitária, 05314-970, São Paulo, São Paulo, Brazil
}

(Received 23 October 2012; revised manuscript received 5 December 2012; published 10 June 2013)

\begin{abstract}
We consider a family of two-dimensional nonlinear area-preserving mappings that generalize the Chirikov standard map and model a variety of periodically forced systems. The action variable diffuses in increments whose phase is controlled by a negative power of the action and hence effectively uncorrelated for small actions, leading to a chaotic sea in phase space. For larger values of the action the phase space is mixed and contains a family of elliptic islands centered on periodic orbits and invariant Kolmogorov-Arnold-Moser (KAM) curves. The transport of particles along the phase space is considered by starting an ensemble of particles with a very low action and letting them evolve in the phase until they reach a certain height $h$. For chaotic orbits below the periodic islands, the survival probability for the particles to reach $h$ is characterized by an exponential function, well modeled by the solution of the diffusion equation. On the other hand, when $h$ reaches the position of periodic islands, the diffusion slows markedly. We show that the diffusion coefficient is scaling invariant with respect to the control parameter of the mapping when $h$ reaches the position of the lowest KAM island.
\end{abstract}

DOI: 10.1103/PhysRevE.87.062904

PACS number(s): 05.45.Pq, 05.45.Tp

\section{INTRODUCTION}

Characterizing mixed regular-chaotic phase space remains one of the most challenging open problems in Hamiltonian dynamics. When there is periodic forcing, the level of chaoticity is typically controlled by the ratio of the intrinsic and extrinsic time scales, a function of phase space variables. For example, the family of maps

$$
T:\left\{\begin{array}{l}
J_{n+1}=\left|J_{n}-\epsilon \sin \left(2 \pi \theta_{n}\right)\right|, \\
\theta_{n+1}=\left[\theta_{n}+J_{n+1}^{-\gamma}\right] \quad(\bmod 1),
\end{array}\right.
$$

for action $J$ and angle $\theta$, describes a large class of systems, including relevant applications for plasma physics for $\gamma=$ 2 [1]. For $\gamma=3 / 2$ it describes the diffusion of the orbital parameters of comets due to the periodic motion of Jupiter $[2,3]$. For this problem the action variable corresponds to the energy of the comets and the second equation recovers Kepler's third law, and because of this it is called the Kepler map. This value of $\gamma$ recovers the dynamics of a wave packet [1] too. For $\gamma=1$ Eq. (1) describes a particle moving between a vibrating and a fixed plate [4]. This case recovers the one-dimensional Fermi-Ulam accelerator model where a particle is confined to bounce between two rigid walls, suffering elastic collisions. The action variable represents the velocity of the particle and the angle variable denotes the phase of the moving wall. $\gamma=1$ recovers also the periodically corrugated waveguide [5]. The model consists in considering a light ray which is specularly reflected by two reflective surfaces. Here the action variable corresponds to the trajectory of the light ray, while the angle variable corresponds to the angle formed between the trajectory of the light and the reflection from the corrugated surface. For $\gamma=1 / 2$, some dynamical properties for a time-dependent potential well $[6,7]$ are recovered. This model consists of a classical particle confined inside a box of an infinitely deep potential which contains an oscillating square well in the middle. It assumes the bottom moves periodically in time according to a cosine function. The action variable represents the energy of the particle while the angle corresponds to the phase of the oscillations. For $\gamma=-1$ a particle bouncing on a vibrating plate [8] is recovered. The model describes the problem of a classical particle bouncing elastically from a periodically time-varying wall in the presence of a constant gravitational field. $\gamma=-1$ recovers also the Chirikov standard map [9]. This model describes a system perturbed by a sequence of pulses (kicks). For clarity we will henceforth assume that $\gamma>0$, although the case $\gamma<0$ can be treated similarly. The time-scale separation is given by $J^{-\gamma}$ so that when $J \approx 1$ there is mixed phase space, while for $J \ll 1, \theta$ is effectively random, leading to strongly chaotic diffusion of $J$, which can be described analytically. Here we develop an open systems approach as a sensitive probe of both chaotic and mixed regions, and demonstrate scale invariance of the diffusion coefficient with respect to the control parameter $\epsilon$.

A powerful technique for analyzing dynamical systems $[10,11]$ involves placing an absorbing "hole" when $J>h$ for some hole parameter $h$ while specifying the distribution of initial conditions, hence defining the ("survival") probability $P(n)$ that the action will remain less than $h$ until $n$ collisions have occurred. This provides a sensitive test of the diffusion of the action variable. In the strongly chaotic regime, $P(n) \sim$ $e^{-A n}$ with an escape rate $A$ that can be predicted by solving the diffusion equation with appropriate initial and boundary conditions and without any free parameters. The relation of transport (for example diffusion) coefficients to the escape rate $A$ in molecular systems was introduced as "the escape rate formalism" $[12,13]$. Here the diffusion is in phase space, and furthermore we predict the full time dependence $P(n)$.

As the action rises, periodic motion is observed, leading to the existence of Kolmogorov-Arnold-Moser (KAM) islands. For even higher action a set of invariant KAM curves (called invariant spanning curves or rotationally invariant circles) is 
present in the phase space, limiting the unbounded growth of the action. Due to the existence of periodic islands, the orbits may spend some time trapped around the regular regions. This temporary trapping, also known as stickiness, affects the diffusion coefficient, which markedly changes at the position of the periodic islands. The relevant scale for the onset of stickiness is the location of the lowest stable island $J_{\mathrm{LSI}}$. In this paper we show that the sticky diffusion process is scale invariant and the scaled diffusion coefficient $D / \epsilon^{2}$ calculated using the open systems approach is a universal function of the scaled hole height $h / J_{\text {LSI }}$.

This paper is organized as follows. In Sec. II we study an analytical approach for the diffusion coefficients of physical systems and study the transport properties. In Sec. III we discuss transport properties and results. Finally, in Sec. IV we present the final discussion.

\section{DIFFUSION COEFFICIENT}

We now consider the dynamics in the chaotic sea for small values of $J$. Due to the lack of correlations of the angle variable, the increments in $J$ are well described using a Central Limit Theorem (CLT) [14,15], so that over many time steps, the distribution of displacements is Gaussian with a variance proportional to the number of steps. Sufficient conditions for a CLT to hold are that $\left\{X_{n}\right\}_{n=1}^{\infty}$ is a sequence of independent and identically distributed (IID) real-valued random variables such that (i) the mean $\left\langle X_{n}\right\rangle=0$ and (ii) $\left\langle X_{n}^{2}\right\rangle<\infty$ [16-18]. In Eq. (1) we see that the angles diverge in the limit of vanishing action and are thus expected to be independent and uniformly distributed on $[0,1)$. The increment of $J$ in Eq. (1) and also in many physical problems in the literature [19] is given by $\Delta_{n}=J_{n+1}-J_{n}=-\epsilon \sin \left(2 \pi \theta_{n}\right)$, and is also IID. Thus it is possible to observe that $\left\langle\Delta_{n}^{2}\right\rangle=\int_{0}^{1} \epsilon^{2} \sin ^{2}(2 \pi \theta) d \theta=\epsilon^{2} / 2$. Therefore the analytical diffusion coefficient is given by $D=$ $\left\langle\Delta^{2}\right\rangle / 2=\epsilon^{2} / 4$, and hence $D / \epsilon^{2}=1 / 4$. We have ignored the absolute value signs here for $J<\epsilon$; their effect is to ensure that $J$ remains positive, i.e., there is a rigid wall in the diffusion process at $J=0$.

Over many iterations, we may consider the continuum process given by the diffusion equation

$$
\frac{\partial u}{\partial n}=D \frac{\partial^{2} u}{\partial J^{2}}
$$

where $u$ is the probability density function. The boundary conditions are given by $d u / d J(0, n)=0$ (the rigid wall, above) and $u(h, n)=0$ (escape through the hole). This problem may be solved by separation of variables, $u(J, n)=$ $X(J) T(n)$, where the boundary conditions give $X(J)=$ $\cos [J \pi(k+1 / 2) / h]$, with $k=0,1,2,3, \ldots$ Substituting for $u(J)$ in Eq. (2), we obtain the solution of the diffusion equation,

$u(J, n)=\sum_{k=0}^{\infty} a_{k} \cos \left[\frac{J \pi\left(k+\frac{1}{2}\right)}{h}\right] \exp \left[\frac{-D n \pi^{2}\left(k+\frac{1}{2}\right)^{2}}{h^{2}}\right]$.

Finally, the unknown coefficients $a_{k}$ are determined by the initial conditions. Here we start all the particles near $J=0$, so that $u(J, 0)=\delta(J)$. Equating this with $a_{k} \cos [J \pi(k+1 / 2) / h]$ as required for Eq. (3), multiplying

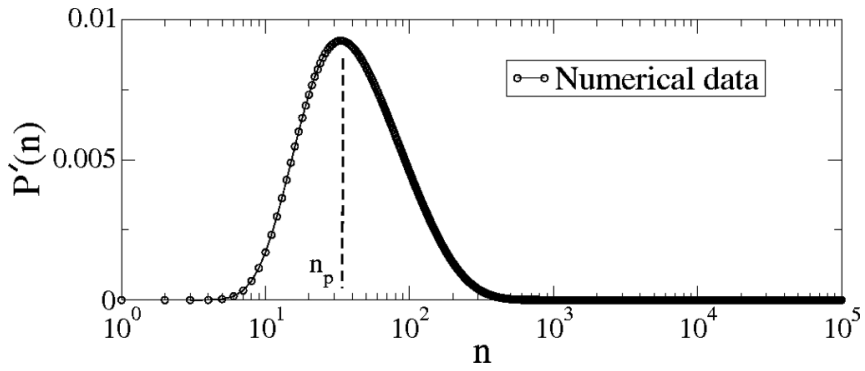

FIG. 1. Plot of $P^{\prime}$ vs $n$ considering $h=10$ and $D=0.5$.

both sides by $\cos [J \pi(m+1 / 2) / h]$, and integrating as a function of $J$ over $[0, h]$ yields

$u(J, n)=\frac{2}{h} \sum_{k=0}^{\infty} \cos \left[\frac{J \pi\left(k+\frac{1}{2}\right)}{h}\right] \exp \left[\frac{-D n \pi^{2}\left(k+\frac{1}{2}\right)^{2}}{h^{2}}\right]$,

which obeys Eq. (2), in both boundary conditions and the initial conditions. Integrating Eq. (4) as a function of $J$ in the range of $[0, h]$, the survival probability is given by

$$
P(n)=\frac{2}{\pi} \sum_{k=0}^{\infty} \frac{\sin \left[\pi\left(k+\frac{1}{2}\right)\right]}{k+\frac{1}{2}} \exp \left[\frac{-D n \pi^{2}\left(k+\frac{1}{2}\right)^{2}}{h^{2}}\right] .
$$

The negative of the derivative of $P(n)$ with respect to $n$, i.e., $P^{\prime}(n)=d P(n) / d n$, furnishes the histogram of frequency for the number of particles that escaped at a time $n$. A plot of $P^{\prime}(n)$ vs $n$ is shown in Fig. 1.

We see from this figure that for short $n$ there is a regime of growth of the histogram of frequency. It then reaches a maximum at $n_{p}$ and decreases later on for large $n$. Few particles can travel to $h$ and so escape at short times. The peak denotes the typical $n$ where the majority of the ensemble reaches $h$. At late times there are few particles remaining, and so the number that escape decreases. Considering Fig. 1 together with the expression of $P^{\prime}(n)$, we see for high values of $h$ that the single term $k=0$ dominates and so $\exp \left[-D n \pi^{2} /\left(4 h^{2}\right)\right] \cong$ $\exp [-A n]$. The diffusion coefficient is written as

$$
D=4 h^{2} A / \pi^{2} \text {. }
$$

The coefficient $A$ may be obtained by fitting an exponential to the histogram of frequency $P^{\prime}(n)$ vs $n$ for the decaying curve after the peak and before the final tail of the curve (which may not be exponential due to the stickiness).

\section{TRANSPORT PROPERTIES AND RESULTS}

The above results are very general, applying to any system modeled by the diffusion equation in this way. We now consider more specifically the family of mappings given in Eq. (1). The control parameters are $\epsilon$ and $\gamma$. For $\epsilon=0$ the system is integrable while it is mixed for any $\epsilon \neq 0$. The mapping is area preserving since the determinant of the Jacobian matrix is equal to 1 . The relevance of particular values of $\gamma$ is given in the Introduction. The phase space generated from the mapping (1) is shown in Fig. 2 for the control parameters (a) $\epsilon=10^{-3}$ and $\gamma=1 / 2$ and (b) $\epsilon=10^{-2}$ and $\gamma=1$. One sees that the phase space is mixed, with strong 

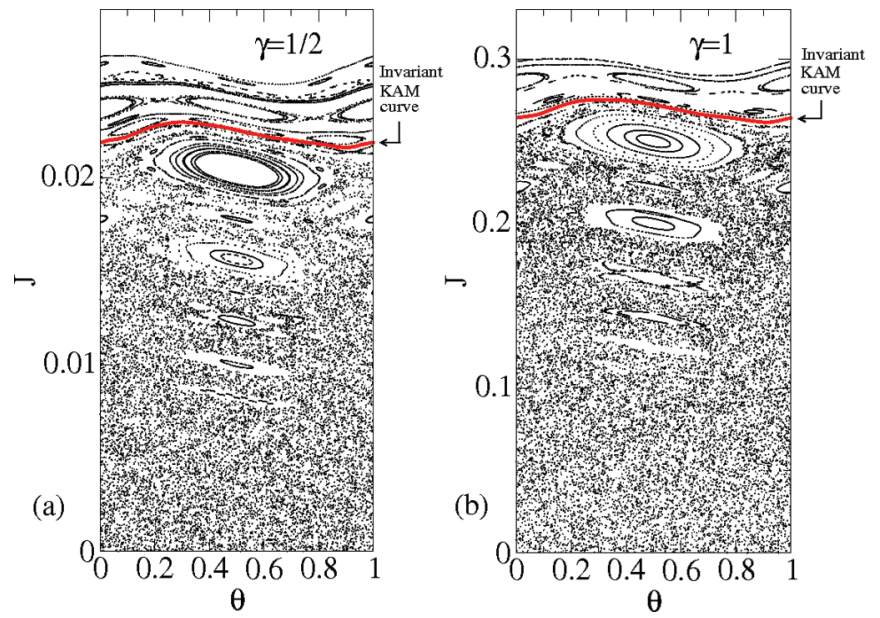

FIG. 2. (Color online) Phase space using (a) $\epsilon=10^{-3}$ and $\gamma=$ $1 / 2$; (b) $\epsilon=10^{-2}$ and $\gamma=1$.

chaos at small $J$, a set of KAM islands that are surrounded by a chaotic sea that is limited by a set of invariant KAM curves.

The size of the chaotic sea can be estimated using the position of the lowest invariant KAM curve. As discussed in [20], near such a curve, the action can be approximated as $J_{n+1} \cong J^{*}+\Delta J_{n+1}$ where $J^{*}$ is a typical value along the curve and $\Delta J_{n+1}$ is a small perturbation of $J^{*}$. Using this approximation and making a connection with the Chirikov standard map [9], which has a transition from local to global chaos at $K=0.9716 \ldots$. the first invariant KAM curve can be described as $J^{*} \cong\left[\frac{2 \pi \gamma \epsilon}{0.9716 \ldots}\right]^{1 /(1+\gamma)}$. The diffusion equation given by (2) and the diffusion coefficient obtained from Eq. (6) can also be well applied for the chaotic region of the phase space generated by the mapping (1) below the lowest action KAM island. When the islands of stability are considered, local trapping may be observed. This stickiness regime affects directly the transport of particles in the phase space, leading to a marked reduction of the diffusion coefficient $D$ (as we shall see in Fig. 4). The understanding of the effects of stickiness in transport is still a major open problem.

The boundary conditions in the phase space are defined by the lowest value of the action (for instance zero) and the position $h<J^{*}$ which defines the upper absorber. To average the quantities, we start with an ensemble of several different initial conditions, the very low initial action of $10^{-3} \epsilon$, and different angles $\theta_{0}$ uniformly distributed along $\theta_{0} \in[0,1]$. If during the dynamics the particle reaches $h$, the simulation is stopped at that time, the number of iterations $n$ needed for the diffusion of the particle until it reaches $h$ is collected from the dynamics, and a simulation with a new initial condition is started with a different angle. The process is repeated until the entire ensemble has been considered. If the maximum length of time of $n=10^{5}$ allowed for the dynamics is reached, we consider that the particle has not escaped (it may escape later in a longer run) and a new initial condition is used.

It is known that when the escape is considered in a region where only chaos is present, the survival probability of an ensemble of particles moving in such a region is described by an exponential decay (see, for instance, $[21,22]$ ). The existence of periodic regions in the phase space, including islands of
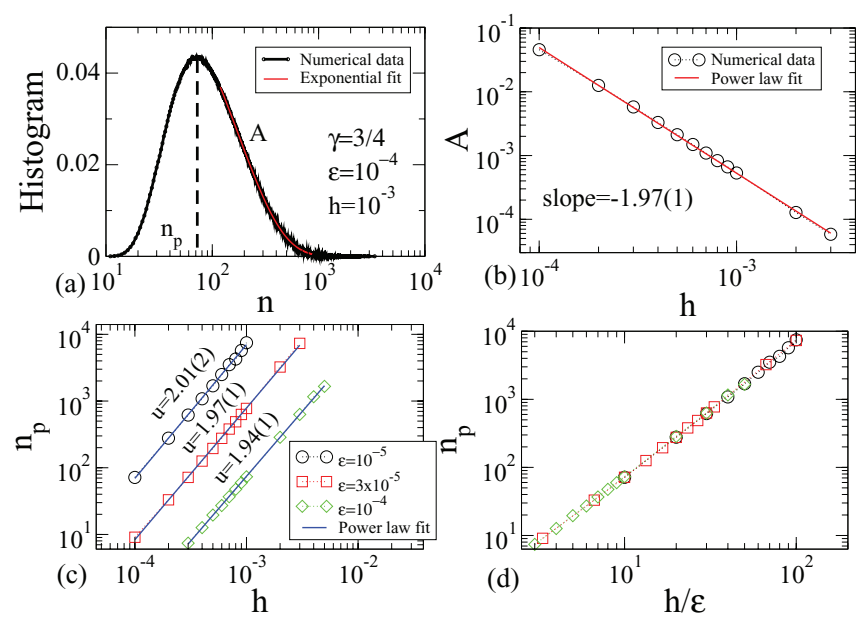

FIG. 3. (Color online) For $\gamma=3 / 4$ we have (a) a plot of the histogram as a function of $n_{p}$, where we highlight how to find $n_{p}$; (b) $A$ vs $h$, where the slope is equal to $-1.97(1)$; (c) $n_{p}$ vs $h$ for different values of $\epsilon: \epsilon=10^{-5}, \epsilon=3 \times 10^{-5}$, and $\epsilon=10^{-4}$; and (d) $n_{p}$ vs $h / \epsilon$.

stability and invariant tori, leads to local trapping due to stickiness [23-25] and consequently to anomalous diffusion [26], transforming the decay of the survival probability into a slower regime that may include either a power law $[27,28]$ or even a stretched exponential [29]. In our simulations, the position $h$ (the upper boundary for escape) was varied. For values of $h$ below the stability regions, an exponential decay describes the survival probability well, while a slower decay is observed when periodic islands are present. Figure 3(a) shows a plot of the histogram of frequency considering the parameters $\gamma=3 / 4$ and $\epsilon=10^{-4}$, although any other set of control parameters generates a similar plot, so that the behavior seems to be generic for any $\gamma>0$ and $\epsilon \neq 0$. The parameter $A$ used in Eq. (6) is obtained by a numerical fitting of the decay of the curve after reaching the peak at $n_{p}$ and before the final tail (where fluctuations due to the size of the ensemble or the size of the bins have influence). Figure 3(b) shows a plot of $A$ vs $h$ and a power law fitting gives the slope as $-1.97(1) \cong-2$, in good agreement with Eq. (6). The peak of the histogram is also sensitive to the position of $h$. It moves to the right in Fig. 3(a) when the position of $h$ is raised in the vertical direction and moves to the left when $h$ moves down in the phase space. This behavior is indeed expected because the higher $h$ becomes, the larger the value of $n$ to which the mapping (1) must be iterated for the particle to reach $h$. A plot of $n_{p}$ vs $h$ is shown in Fig. 3(c). If the position $h$ is rescaled as $h \rightarrow h / \epsilon$, the different curves of $n_{p}$ obtained for different $\epsilon$ overlap onto a single plot as shown in Fig. 3(d).

Let us now discuss our numerical results obtained for the diffusion coefficient. From numerical simulations, we obtained different values for the coefficient $A$ and Eq. (6) was evaluated. The diffusion coefficient is shown in Fig. 4(a) for different control parameters $\epsilon$ and $\gamma$. Different values of the control parameter $\epsilon$ generate different values for $D$ in agreement with $D / \epsilon^{2}=1 / 4$. We notice that $D$ is almost constant for a large range of $h$ until it suddenly suffers a marked decrease. The decrease happens because the position $h$ reached the lowest 

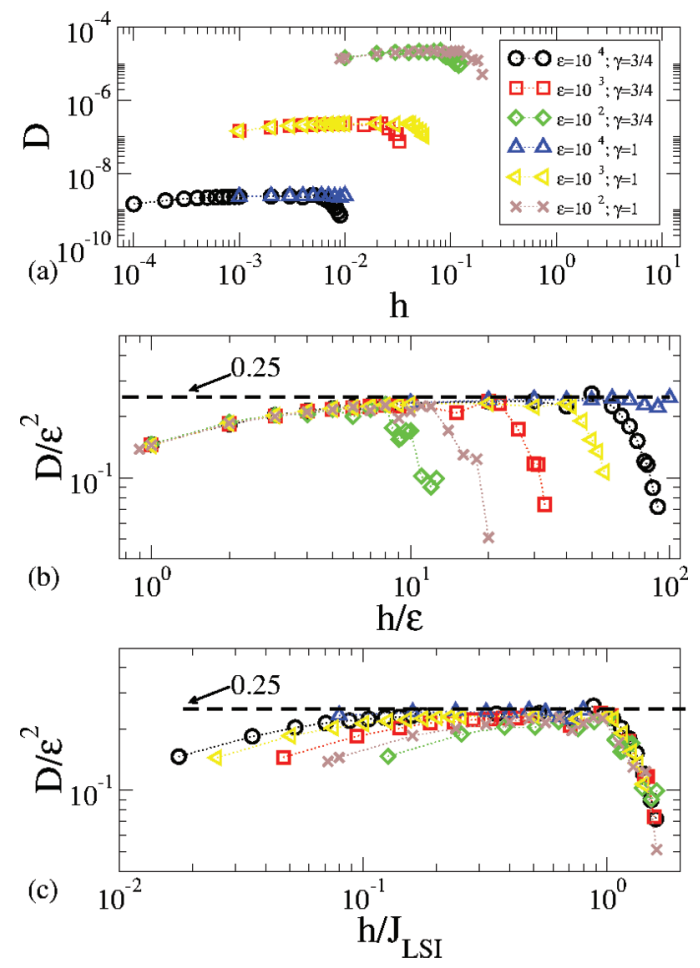

FIG. 4. (Color online) (a) $D$ vs $h$ for different values of $\epsilon$ and $\gamma$. (b) After a rescaling on the axis, the curves collapsed for small values of $h$. (c) After a properly rescaling on the axis, all curves collapsed for high values of $h$.

action KAM island. From fixed point stability analysis we found that the last period-1 fixed point, generating the last period-1 island, is located at

$$
J_{\text {LSI }} \cong\left[\frac{\gamma \pi \epsilon}{2}\right]^{[1 /(1+\gamma)]},
$$

where the index LSI stands for lowest stable KAM island. We see that there are two different types of scaling for the behavior of $D$. The first scaling is observed for very small values of $h$. In this region, there is presumably a breakdown of the continuum approximation used in the diffusion equation since $h$ comes close enough to the scale of the variation of $\epsilon$. Figure 4(b) shows the behavior of $D$ for the rescaling $D \rightarrow D / \epsilon^{2}$ and $h \rightarrow h / \epsilon$. We see clearly that all curves of $D$ obtained for different control parameters as shown in Fig. 4(a) overlap each other for low values of $h / \epsilon$ but differ for large $h$. A striking result is obtained when the horizontal axis is rescaled with respect to the position of the lowest stable KAM island as given by Eq. (7), i.e., $h \rightarrow h / J_{\mathrm{LSI}}$, as shown in Fig. 4(c). In addition we see that despite the small difference of $D / \epsilon^{2}$ for different control parameters $\epsilon$ considering small $h$, the diffusion coefficient suffers a sudden change when the position $h$ reaches periodic KAM islands. Because of the periodic regions, the particles may suffer temporary trapping due to stickiness and its influence on the transport along the phase space. At that point normal diffusion is not observed anymore; consequently the CLT is not applicable in this domain.

\section{FINAL DISCUSSION}

We have obtained analytically the diffusion coefficients for many physical systems and studied the transport properties for a set of classical particles for a family of two-dimensional Hamiltonian mappings. The curves of the histograms were obtained numerically and an exponential fit was made. Our results show that for low values of the hole presumably a breakdown of the continuum approximation occurs as the hole comes close to the scale of the variations $\epsilon$. For high values of the hole (near the KAM islands and spanning KAM curve), we showed that the hole also comes close to the scale of the variations $\epsilon$. The present procedure can be applied to many other different systems where transport properties are observed. In future it would be interesting to investigate whether a modified, perhaps fractional, diffusion equation could apply to the variation of $J$ in the sticky region, and whether the same kind of stickiness is present for the impenetrable lowest invariant spanning curve. Many of the applications, including the Kepler (now Coulomb) problem and motion in the presence of a vibrating wall, occur in the microscopic domain, where quantum effects dominate.

\section{ACKNOWLEDGMENTS}

J.A.O. thanks CAPES and PROPe/UNESP, C.P.D. and D.R.C. are grateful to FAPESP, and E.D.L. thanks FAPESP, CNPq, and FUNDUNESP, Brazilian agencies, for support. This research was supported by resources supplied by the Center for Scientific Computing (NCC/GridUNESP) of the São Paulo State University (UNESP).
[1] G. M. Zaslavsky, R. D. Sagdeev, D. A. Usikov, and A. A. Chernikov, Weak Chaos and Quasi-Regular Patterns (Cambridge University Press, Cambridge, 1991).

[2] G. M. Zaslavsky, Hamiltonian Chaos and Fractional Dynamics (Oxford University Press, Oxford, 2005).

[3] I. I. Shevchencko, New Astron. 16, 94 (2011).

[4] M. A. Lieberman and A. J. Lichtenberg, Phys. Rev. A 5, 1852 (1972).

[5] G. A. Luna-Acosta, J. A. Mendez-Bermudez, and F. M. Izrailev, Phys. Rev. E 64, 036206 (2001); Phys. Lett. A 274, 192 (2000).
[6] G. A. Luna-Acosta, G. Orellana-Rivadeneyra, A. MendozaGalván, and C. Jung Chaos, Solitons Fractals, 12, 349 (2001).

[7] J. L. Mateos, Phys. Lett. A 256, 113 (1999).

[8] L. D. Pustylnikov, Trans. Moscow Math. Soc. 2, 1 (1978).

[9] A. J. Lichtenberg and M. A. Lieberman, Regular and Chaotic Dynamics (Springer, New York, 1992), p. 38.

[10] L. A. Bunimovich and C. P. Dettmann, Europhys. Lett. 80, 40001 (2007).

[11] E. G. Altmann, S. E. Portela, and Tamas Tel, Rev. Mod. Phys. 85, 869 (2013).

[12] P. Gaspard and G. Nicolis, Phys. Rev. Lett. 65, 1693 (1990). 
[13] J. R. Dorfman and P. Gaspard, Phys. Rev. E 51, 28 (1995).

[14] W. J. Adams, The Life and Times of the Central Limit Theorem, 2nd ed. (American Mathematical Society, Providence, RI, 2009).

[15] H. Fischer, History of the Central Limit Theorem (Springer, New York, 2011).

[16] I. Eliazar and J. Klafter, Chem. Phys. 370, 290 (2010).

[17] H. J. Hilhorst, Braz. J. Phys 39, 371 (2009).

[18] S. Umarov, C. Tsallis, and S. Steinberg, Milan J. Math. 76, 307 (2008).

[19] G. M. Zaslavsky, Phys. Rep. 371, 461 (2002).

[20] E. D. Leonel, J. A. de Oliveira, and F. Saif, J. Phys. A 44, 302001 (2011).

[21] C. F. F. Karney, Physica D 8, 360 (1983).
[22] M. F. Demers and L.-S. Young, Nonlinearity 19, 377 (2006)

[23] V. A. Avetisov and S. K. Nechaev, Phys. Rev. E 81, 046211 (2010).

[24] D. L. Shepelyansky, Phys. Rev. E 82, 055202(R) (2010).

[25] G. Cristadoro and R. Ketzmerick, Phys. Rev. Lett. 100, 184101 (2008).

[26] S. S. Abdullaev and K. H. Spatschek, Phys. Rev. E 60, R6287 (1999).

[27] H. Henry et al., Chaos 9, 381 (1999).

[28] N. Buric, A. Rampioni, G. Turchetti, and S. Vaienti, J. Phys. A 36, L209 (2003).

[29] C. P. Dettmann and E. D. Leonel, Physica D 241, 403 (2012). 